\title{
Diluted Josephson-junction arrays in a magnetic field: Phase coherence and vortex glass thresholds
}

\author{
Mourad Benakli \\ Condensed Matter Physics Group, International Centre for Theoretical Physics, 34100 Trieste, Italy \\ Enzo Granato \\ Laboratório Associado de Sensores e Materiais, Instituto Nacional de Pesquisas Espaciais, 12225-São José dos Campos, SP, Brazil \\ Subodh R. Shenoy \\ Condensed Matter Physics Group, International Centre for Theoretical Physics, 34100 Trieste, Italy \\ Marc Gabay \\ Laboratoire de Physique des Solides Laboratoire associé au CNRS, Université de Paris-Sud, Bâtiment 510, 91405 Orsay Cedex, France
}

(Received 6 October 1997)

\begin{abstract}
The effects of random dilution of junctions on a two-dimensional Josephson-junction array in a magnetic field are considered. For rational values of the average flux quantum per plaquette $f$, the superconducting transition temperature vanishes, for increasing dilution at a critical value $x_{S}(f)$, while the vortex ordering remains stable up to $x_{\mathrm{VL}}>x_{S}$, far below the value $x_{p}$ corresponding to the geometric percolation threshold. For $x_{\mathrm{VL}}<x<x_{p}$, the array behaves as a zero-temperature vortex glass. Numerical results for $f=1 / 2$ from defect energy calculations are presented and are consistent with this scenario. [S0163-1829(98)00818-2]
\end{abstract}

Vortex-glass states in disordered three-dimensional superconductors have been the focus of much recent interest. ${ }^{1-5}$ In the absence of screening, they are believed to have a true superconducting phase, with vanishing linear resistivity, at finite temperatures. By contrast, in two dimensions, vortexglass models ${ }^{2}$ and experiments on superconducting films ${ }^{5}$ show that vortex-glass order is destroyed at any finite temperature with a nonzero but exponentially small resistivity. This zero-temperature vortex glass can be characterized by a thermal correlation length exponent $\nu_{T}$ which determines, for example, the current density scale, $J_{\mathrm{nl}} \sim T^{1+\nu_{T}}$, where nonlinear behavior shows up in the current-voltage characteristics. $^{2,5}$ Recent estimates give $\nu_{T} \sim 2$ for various vortex glass models. ${ }^{2}$

Randomly diluted Josephson-junction arrays (JJA's) have been used to model disordered superconductors. ${ }^{3,6,7}$ In zero field, the superconducting transition temperature vanishes at

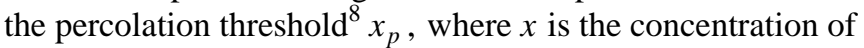
diluted junctions. For $x>x_{p}$ there are only uncoupled finite clusters and long-range phase coherence is destroyed. At $x_{p}$, the infinite percolating cluster shows up in the scaling behavior of the dynamic conductivity ${ }^{6}$ and nonlinear resistivity. ${ }^{7}$ In the presence of an external field, a diluted JJA is an experimentally controllable model to investigate phase coherence and vortex glasses in two dimensions. For rational values of the flux quantum per unit cell $f$, an ordered $(x=0)$ JJA has a ground state consisting of a periodic pinned vortex lattice, with additional discrete symmetries resulting from commensurability effects. ${ }^{1,9}$ The melting of this vortex lattice at a temperature $T_{\mathrm{VL}}$, driven by domain-wall excitations, competes with the superconducting transition at $T_{S}$ driven by the Kosterlitz-Thouless vortex unbinding. For $f=1 / 2$, these transitions either coincide or have very close transition temperatures, ${ }^{1} T_{\mathrm{VL}} \gtrsim T_{S}$. Similar behavior is expected for other low rational values of $f$. In presence of randomdilution disorder, two natural questions arise: (i) are there two dilution thresholds, $x_{S}$ and $x_{\mathrm{VL}}$, for phase coherence and vortex-lattice order, respectively? Does a vortex-glass phase occur over a significant range $x>x_{\mathrm{VL}}$ ?

In this work, we argue that for rational values of $f$, the superconducting transition temperature of the array vanishes, for increasing dilution, at a critical value $x_{S}(f)$. The vortexlattice ordering remains stable up to $x_{\mathrm{VL}}(f)>x_{S}(f)$ but both values are much below the value $x_{p}$ corresponding to the geometric percolation threshold. For $x_{\mathrm{VL}}<x<x_{p}$ there is a zero-temperature vortex glass. These features are verified numerically for $f=1 / 2$, using a bond-diluted frustrated $X Y$ model on a triangular lattice, and extensive zero-temperature calculations. Domain-wall energy calculations give an estimate of a wide range, $x_{\mathrm{VL}}<x<x_{p}$, for a zero-temperature vortex glass below the geometrical percolation threshold $x_{p}$ $=0.652$. We find $x_{S}=0.14(1)$ and $x_{\mathrm{VL}}=0.17(1)$ consistent with the proposed scenario. In the vortex-glass phase, $\nu_{T}$ $\sim 1.9$, as estimated from the size dependence of defect energies excitations. Interestingly enough, this estimate is very close to the value obtained for the gauge-glass model $^{2}$ which may suggest a common universality class.

We consider a two-dimensional Josephson-junction array in a magnetic field $B$ described by the Hamiltonian of a frustrated $X Y$ model

$$
H=-\sum_{\langle i j\rangle} J_{i j} \cos \left(\theta_{i}-\theta_{j}-A_{i j}\right),
$$

where $\theta_{i}$ is the phase of the condensate wave function in a grain at site $i$ and $J_{i j}$ is the Josephson coupling. The summation is taken over all nearest neighbors of a regular reference lattice. The dimensionless line integral of the vector 


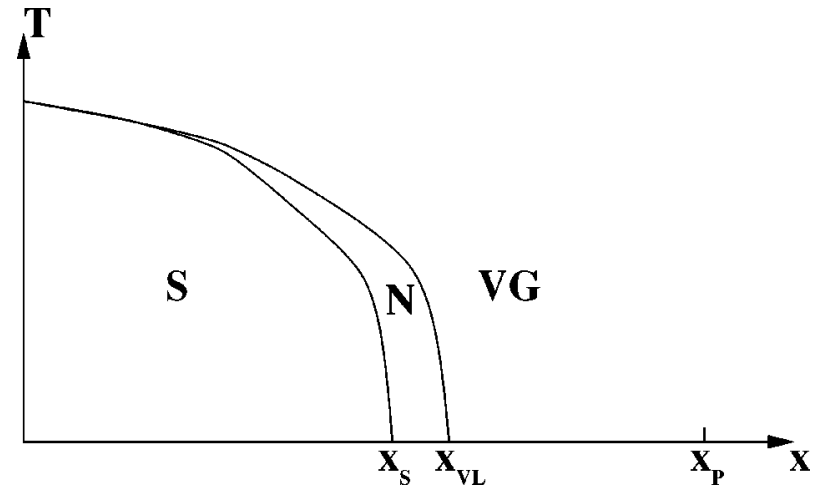

FIG. 1. Schematic phase diagram of a diluted JJA as a function of temperature $T$ and dilution $x$, for an average rational frustration $f$, showing a superconducting phase (S), a normal ordered vortexlattice phase $(\mathrm{N})$, and a vortex-glass phase (VG). A single transition is assumed at $x=0$. If the transitions are already separated at $x$ $=0$, dilution would further increase the separation. At $T=0$, a vanishing linear resistance is expected for $x<x_{p}$ due to vortex pinning.

potential $A_{i j}$ about each elementary reference-lattice plaquette of area $S$ is $\Sigma_{p} A_{i j}=2 \pi f$, where the frustration parameter $f=B S / \Phi_{o}$ measures the number of flux quanta $\Phi_{o}$ per plaquette. A bond-dilution concentration $x$ corresponds to $J_{i j}$ being zero or $J$, with probabilities $x$ and $1-x$, respectively. Since any closed loops of nonzero bonds $J$ have an area which is an integer multiple of the elementary area $S$, the properties of this model are periodic in $f$ with period 1 , and it is therefore sufficient to consider $0<f<1$.

For $f=0$, the Hamiltonian reduces to the standard diluted $X Y$ model, which is known to be superconducting for ${ }^{8} x$ $<x_{p}$. When $f \neq 0$, there must also be a threshold $x_{\mathrm{VL}}$ for vortex-lattice disordering ${ }^{4}$ below the percolation threshold, $x_{\mathrm{VL}}<x_{p}$. In the undiluted case $x=0$, the ground state for rational $f=p / q(q \geqslant 2)$ consists of a pinned vortex lattice ${ }^{9}$ with a $q \times q$ unit cell. For small dilution $x \ll x_{\mathrm{VL}}$, the longrange order of the vortex lattice persists, provided an infinite cluster of these cells exists. Since $x_{\mathrm{VL}}(f)$ corresponds roughly to the percolation threshold for cells of size $q \times q$, the percolation threshold for $q \times q$ cell dilution is reached much below the unit bond-dilution threshold. Alternatively, long-range order of the vortex lattice requires connectivity over at least $q$ bonds, as in bootstrap percolation, ${ }^{10}$ which is known to lead to a percolation threshold below the unit bond percolation. Since vortex-lattice disordering leads to suppression of phase coherence, ${ }^{1} x_{\mathrm{VL}}$ is an upper bound for the superconducting threshold $x_{S}$. This implies that the transition temperature should vanish at an $x_{S} \leqslant x_{p}$, and that the thresholds are as illustrated in Fig. 1. At least for low-order rational values of $f$, we would expect $x_{S}\left(f^{\prime}\right)<x_{S}(f)$ if $f^{\prime}$ $<f$ since $f^{\prime}$ requires a higher connectivity. For $x_{\mathrm{VL}}(f)<x$ $<x_{p}$, there is no long-range order, and this phase should correspond to a two-dimensional vortex glass, where a true phase transition is known to occur only at $T=0 .{ }^{2,11} \mathrm{An}$ intervening glass phase near percolation threshold is also expected from mean-field theory. ${ }^{3}$ This phase can be characterized $^{2}$ by a critical exponent $\theta$ that determines how low-energy excitations $\Delta E(L)$ from the ground state behave at long length scales $L$. For a $T=0$ vortex glass $\Delta E \sim L^{\theta}$, with $\theta<0$, and thermal excitations of scale $\xi \sim T^{-\nu_{T}}$ destroy the glass order at any finite temperature, leading to an identification of the thermal correlation length exponent as $\nu_{T}$ $=1 /|\theta|$. Our numerical results for $f=1 / 2$, described below, are consistent with this behavior, and provide an estimation of $\nu_{T}$. In absence of thermal fluctuations, at $T=0$, vortices are pinned by disorder and a nonlinear response to an applied current is expected leading to a vanishing linear resistance and nonzero critical current for $x<x_{p}$.

We have carried out a detailed numerical study for $f$ $=1 / 2$ at zero temperature, using a bond diluted frustrated JJA on a triangular lattice, where the critical dilution threshold for bond percolation ${ }^{8}$ is $x_{p}=0.652$. For this value of $f$, vortex-lattice ordering can be conveniently described in terms of a $Z_{2}$ chirality order parameter $\left.\chi=\Sigma_{\langle i j\rangle}\right\rangle \theta_{i}-\theta_{j}$ $\left.-A_{i j}\right) /(2 \pi)$, where summation is taken about an elementary plaquette of the actual lattice, and the gauge-invariant phase difference is restricted to the interval $[-\pi,+\pi]$. In the undiluted case, the ground state consists of a pinned vortex lattice corresponding to an antiferromagnetic arrangement of $\chi= \pm 1 / 2$. To study the stability of the ordered phases, we use a defect energy renormalization analysis ${ }^{12}$ at $T=0$. A defect is created in a system of size $L \times L$ by imposing a change in the boundary conditions in one direction. The change $\Delta E(L)$ in the ground-state energy for small systems is calculated for a large number of samples by directly searching for the minimum energy. We used an improved algorithm based on Ref. 13. Typically, 3000 configurations of disorder have been used for each system size. To study both phase coherence and vortex-lattice order, we consider two types of defects: (i) From the energy difference between periodic $E_{p}$ and antiperiodic $E_{a}$ boundary conditions in the phases $\theta_{i}$ we obtain $\Delta E_{1}=E_{a}-E_{p}$, which is a measure of phase coherence, and is related to the renormalized stiffness constant $J(L)=\rho \Delta E_{1} / 2 \pi^{2}$, where $\rho=2 / \sqrt{3}$ is a geometrical factor for the triangular lattice. In the thermodynamic limit, $J$ is finite in the phase-coherent state and vanishes in the incoherent state; (ii) A domain-wall defect energy is obtained as $\Delta E_{2}=E_{r}-E_{p}$, where $E_{r}$ is the ground-state energy with reflected boundary conditions, ${ }^{13}$ corresponding to the energy cost for a domain wall in the vortex lattice. In the presence of disorder, $\Delta E_{1}$ and $\Delta E_{2}$ fluctuate between samples, with a distribution that can be characterized by its moments. Stability of the ground state against thermal fluctuations requires that the average $[\Delta E]$, where [ ] denotes a disorder average, is finite or increases with $L$ for the $U(1)$ and $Z_{2}$ symmetries, respectively. Figure 2 shows the behavior of the $\left[\Delta E_{1}\right]$ as a function of $L$ for increasing dilution. For small $x$, it increases with $L$, indicating the existence of long-range phase coherence. ${ }^{14}$ For sufficiently large $x$ it clearly decreases for increasing $L$, indicating a disordered phase. The change in the behavior yields an estimate of $x_{S}=0.14(1)$. Figure 3 shows a similar plot for $\left[\Delta E_{2}\right]$. The increasing trend with $L$ for small $x$ corresponds to a vortex-lattice ordered phase, which persists for a small but finite range above $x_{S}$. For large $x$, it decreases with $L$, and yields an estimate of $x_{\mathrm{VL}}$ $=0.17(1)$. Thus $x_{\mathrm{VL}}>x_{S}$, as indicated in Fig. 1. The disordered phase for $x_{\mathrm{VL}}<x<x_{p}$ can be regarded as a vortex glass, since it lacks long range order in the vortex lattice.

The stability of the glass phase against thermal fluctuations is determined by the size dependence of the second 


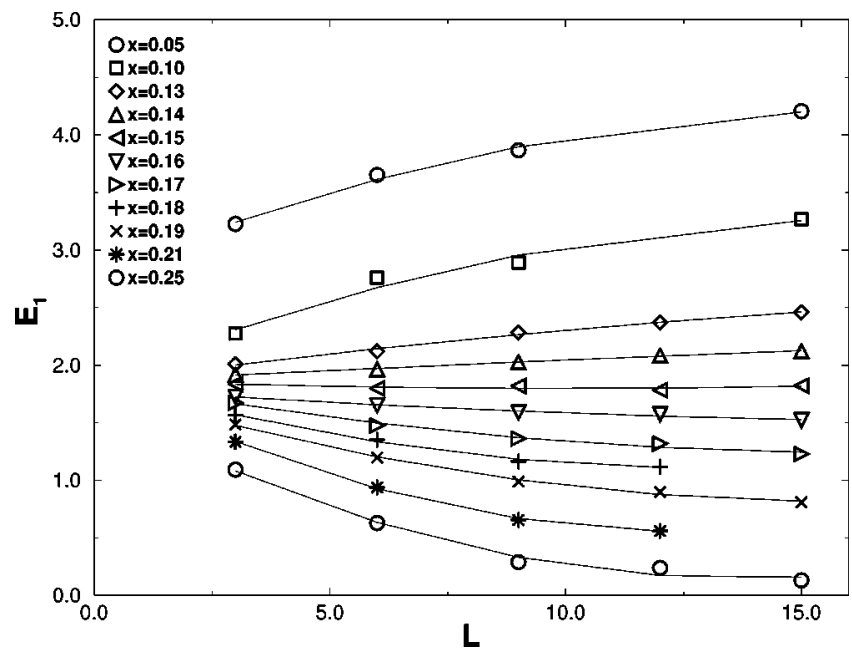

FIG. 2. Finite-size behavior of defect energy $\left[E_{1}\right]$ probing the superfluid density for increasing dilution $x$ and various system sizes $L$. The change in the $L$ dependence determines the threshold $x_{S}$.

moment of the energy excitations $w_{i}=\sqrt{\left[\Delta E_{i}^{2}\right]-\left[\Delta E_{i}\right]^{2}}$ $\propto L^{\theta}$. Here $\theta>0$ indicates a glass phase at nonzero temperature, whereas $\theta<0$ implies that arbitrarily low-energy excitations at long length scales can be thermally excited, destroying the glass phase at any finite temperature. ${ }^{12}$ The size dependence of $w$, for a value of $x=0.3$ in this region ${ }^{15}$ is shown in Fig. 4 and clearly indicates a negative $\theta$ for both $w_{1}$ and $w_{2}$, and so the vortex glass only occurs at $T=0$. The exponent $\nu_{T}=1 /|\theta|$ of the superconducting thermal correlation length $\zeta \propto T^{-\nu_{T}}$ can be estimated from the slope of $w_{1}$ in a log-log plot, giving $\nu_{T} \sim 1$.9. Interestingly enough, this estimate is very close to the value obtained for the gauge-glass model, ${ }^{2}$ suggesting a common universality class, but further data would be necessary to check whether $\nu_{T}$ is $x$ dependent.

At finite temperatures, thermally excited vortices and disorder effects can significantly reduce the ordered phases for $x<x_{\mathrm{VL}}(f)$, since bond dilution introduces correlated randomness in the flux, as in the case of an array with disorder only in the positions of the grains. ${ }^{16}$ Unlike positional disor-

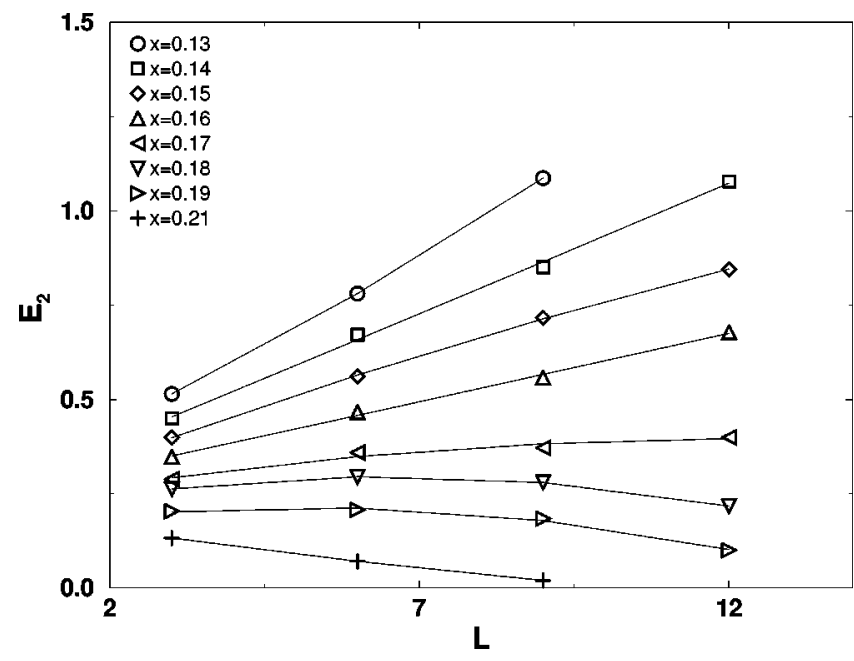

FIG. 3. Finite-size behavior of defect energy $\left[E_{2}\right]$ probing the vortex-lattice stability for increasing dilution $x$ and various system sizes $L$. The change in $L$ dependence determines the threshold $x_{\mathrm{VL}}$.

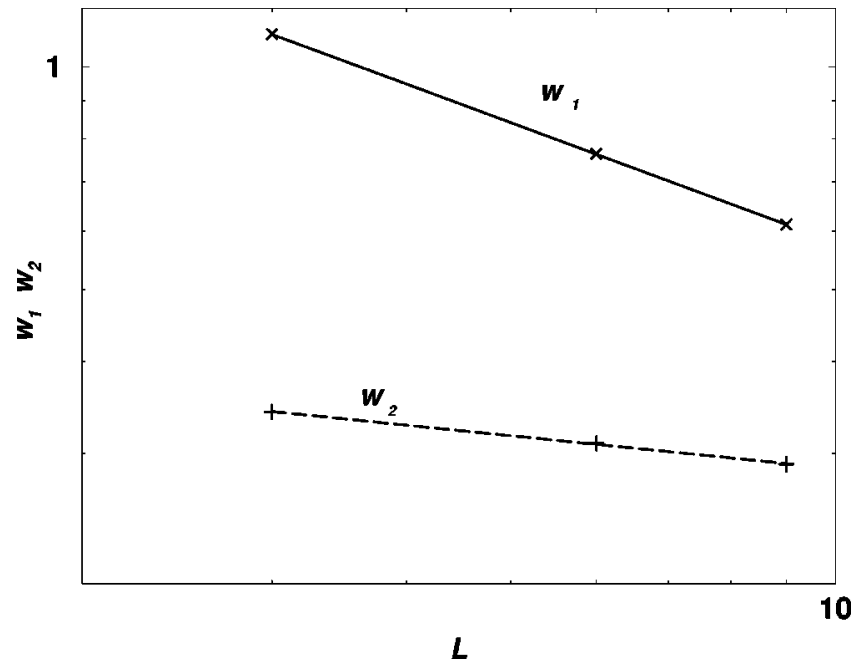

FIG. 4. Finite-size behavior of the second moment of the defect energy distribution $w_{1}$ and $w_{2}$ in the region $x_{\mathrm{VL}}<x<x_{p}$ for $x$ $=0.3$. The negative slope of $\ln w_{1} \times \ln L$ gives an estimate of $1 / \nu_{T}$.

der, random dilution does not explicitly affect the phase difference $\theta_{i}-\theta_{j}$ between two superconducting grains in Eq. (1). Its relevance can be studied through two coupled frustrated $X Y$ models

$$
\begin{aligned}
H= & -\frac{J}{2} \sum_{\langle i j\rangle}\left[\cos \left(\theta_{i}-\theta_{j}-A_{i j}\right)+\cos \left(\phi_{i}-\phi_{j}-A_{i j}-\pi t_{i j}\right)\right] \\
& -h \sum_{i} \cos \left(\theta_{i}-\phi_{i}\right)
\end{aligned}
$$

where $t_{i j}$ is 1 or 0 with probability $x$ and $1-x$, respectively. In the limit $h \rightarrow \infty$, the phases are coupled $\theta_{i}=\phi_{i}$, and the original model in Eq. (1) is recovered. The second term has the same form as the Hamiltonian describing positional disorder in a superconducting array in the presence of magnetic field, ${ }^{16}$ with a particular bimodal distribution of $t_{i j}$. A detailed analysis in the small $h$ limit combined with known $T$ $\neq 0$ results ${ }^{1,9}$ for $x=0$ and the above calculations at $T=0$, suggest the phase diagram of Fig. 1. For coupled $X Y$ models without disorder, ${ }^{16}$ the coupling $h$ renormalizes to large values even when initially small, while the phase transitions can be described in terms of vortices in the average phase variable $\left(\theta_{i}+\phi_{i}\right) / 2$. Guided by this, we consider initially the two $X Y$ models in Eq. (2) to be independent, and consider the particular rational value, $f=1 / 2$, where the relevant excitations, chiral domain walls and vortex charges, are better understood. ${ }^{1,16}$ In this case, the disorder variables act as random bonds on the chiral order parameter $\chi$, and as random dipoles on the vortex charges. If the transition in the pure case is single (simultaneous disordering of the chiral and $X Y$-like variables), the differently acting disorder can thus separate the two transitions with vortex unbinding at temperatures below the chiral transition. ${ }^{16}$ In fact, Monte Carlo simulations for the frustrated $X Y$ model on a square lattice with positional disorder are consistent with the splitting into two transitions. ${ }^{17}$ For the triangular lattice considered here, we have estimated the chiral transition temperature at $x$ $=x_{S}$, where $T_{S}=0$ (Fig. 1), from the peak in the chiral 
susceptibility and found $T_{\mathrm{VL}}=0.27(3)$ which can be compared with the estimated separation ${ }^{20} \Delta T_{c}=0.01$ at $x=0$, if one assumes a double transition, which clearly shows that disorder tends to separate the transitions. The chiral transition is expected to be in the universality class of the random bond Ising model, where recent studies have shown that the specific heat has a broad peak with a very weak $\log -\log (T$ $-T_{c}$ ) divergence but the other exponents remain with the pure Ising model values. ${ }^{18}$ This is consistent with Monte Carlo simulations of the frustrated $X Y$ model on a sitediluted square lattice, ${ }^{19}$ where it is found that the specific heat has a broad peak which does not clearly grow with lattice size, in contrast to the undiluted case which grows almost logarithmically. Even when a finite coupling between the two terms in the Hamiltonian of Eq. (2) is taken into account, the effects of disorder on the chiral order parameter should still remain, since the coupling term should essentially lock equivalent vortices and chiral variables in both phases $\theta_{i}$ and $\phi_{i}$. For other values of $f$, we expect similar qualitative behavior, as illustrated in Fig. 1, but with the chiral transition replaced by the thermal disordering transition of a vortex lattice with a higher-order discrete symmetry.

Experimentally, the vortex-glass phase for $x_{\mathrm{VL}}<x<x_{p}$ could be identified through the change in the current-voltage characteristics ${ }^{5}$ extracting the critical exponent $\nu_{T}$. Another signature would be the disappearance of ordered-phase resistance minima at $f=p / q$ when $x$ is in the $f$-insensitive vortex-glass region $x_{\mathrm{VL}}(f=1 / 2)<x<x_{p}$.

The work of E.G. was supported by ICTP/IAEA and FAPESP (Proc. 97/07250-8).
${ }^{1}$ See articles, in Proceedings of the ICTP Workshop on Josephson Junction Arrays, 1995 [Physica B 222, 253 (1996)].

${ }^{2}$ D. S. Fisher, M. P. A. Fisher, and D. A. Huse, Phys. Rev. B 43, 130 (1991); R. A. Hyman, M. Wallin, M. P. A. Fisher, S. M. Girvin, and A. P. Young, ibid. 51, 15304 (1995); C. Wengel and A. P. Young, ibid. 56, 5918 (1997).

${ }^{3}$ S. John and T. C. Lubensky, Phys. Rev. Lett. 55, 1014 (1985).

${ }^{4}$ T. Giamarchi and P. Le Doussal, Phys. Rev. Lett. 72, 1530 (1994).

${ }^{5}$ C. Dekker, P. J. M. Wöltgens, R. H. Koch, B. W. Hassey, and A. Gupta, Phys. Rev. Lett. 69, 2717 (1992).

${ }^{6}$ D. C. Harris, S. T. Herbert, D. Stroud, and J. C. Garland, Phys. Rev. Lett. 25, 3606 (1991); A.-L. Eichenberger, J. Affolter, M. Willemin, M. Mombelli, H. Beck, P. Martinoli, and S. E. Korshunov, ibid. 77, 3905 (1996).

${ }^{7}$ E. Granato and D. Dominguez, Phys. Rev. B 56, 14671 (1997).

${ }^{8}$ D. Stauffer and A. Aharony, Introduction to Percolation Theory (Taylor and Francis, London, 1992).

${ }^{9}$ S. Teitel and C. Jayaprakash, Phys. Rev. Lett. 51, 1999 (1983).

${ }^{10}$ J. Adler, Physica A 171, 453 (1991).

${ }^{11}$ A superconducting freezing transition may also occur at sufficiently low temperatures and finite cooling rate due to nonequilibrium processes [S. R. Shenoy, Phys. Rev. B 35, 8652 (1987); Physica B 152, 72 (1988)].
${ }^{12}$ A. J. Bray and M. A. Moore, J. Phys. C 17, L463 (1984); M. Cieplak, J. R. Banavar, and A. Khurana, J. Phys. A 24, L145 (1991).

${ }^{13}$ P. Gawiec and D. R. Grempel, Phys. Rev. B 44, 2613 (1991); J. Maucourt and D. R. Grempel, Phys. Rev. Lett. 80, 770 (1998).

${ }^{14}$ This increasing trend, however, is a small size effect. For large $L$, it should scale as $L^{d-2}$.

${ }^{15}$ In a small range $x_{\mathrm{VL}}<x<x_{v *}$, both $w_{2}$ and $\left[E_{2}\right]$ increase with $L$ which might indicate that a chiral glass phase is stable to thermal fluctuations. However, with the limited system sizes used it is not possible to rule out that this small range shrinks to zero for sufficiently large $L$ leading to $x_{\mathrm{VL}}=x_{v *}$. Similar behavior has been found in an Ising model for a varying concentration of antiferromagnetic bonds [N. Kawashima and H. Rieger, Europhys. Lett. 39, 85 (1997)].

${ }^{16}$ E. Granato and J. M. Kosterlitz, Phys. Rev. Lett. 62, 823 (1989); E. Granato, J. M. Kosterlitz, and J. Poulter, Phys. Rev. B 33, 4767 (1986).

${ }^{17}$ D. B. Nicolides, J. Phys. A 24, L231 (1991).

${ }^{18}$ W. Selke, L. N. Shchur, and A. L. Talapov, in Annual Reviews of Computational Physics, edited by D. Stauffer (World Scientific, Singapore, 1994), Vol. 1.

${ }^{19}$ X. C. Zeng, D. Stroud, and J. S. Chung, Phys. Rev. B 43, 3042 (1991).

${ }^{20}$ HJ. Xu and B. W. Southern, J. Phys. A 29, L133 (1996). 